\title{
Bürgeraktien - Ein Beitrag zur Finanzierung kommunaler Unternehmen?
}

Bürgeraktien; Bürgeraktionär; Bürgerbeteiligung; (kommunale) Aktiengesellschaften; (kommunale) Finanzierung; (kommunale) Unternehmen; Repolitisierung; Transparenz

Das Konzept der Bürgeraktie verfolgt zwei Überlegungen: zum einen, ob die Veräußerung von Geschäftsanteilen mittels Kapitalerhöhung an die Bürger die finanzielle Situation von kommunalen Unternehmen verbessern kann; und zum anderen, ob ein Demokratiemoment in der Hauptversammlung des kommunalen Unternehmens installiert wird und die Bürger - als gleichzeitige Miteigentümer und Kunden - an der strategischen Steuerung des Unternehmens beteiligt werden können. Die Bürgerbeteiligung kann mit Sicherheit die Transparenz des Unternehmens zugunsten der Bürger und Kunden erhöhen und das Vertrauen in das Unternehmen stärken.

\section{Die Bürgeraktie als Finanzierungsinstrument für kommunale Unternehmen}

\section{Das Konzept der Bürgeraktie}

Die Bürgeraktie ist ein Anteil am Grundkapital einer kommunalen Aktiengesellschaft im Sinne des $\S 1$ Abs. 2 AktG. Es handelt sich jedoch um ein Wertpapier, welches vorrangig den Personen zum Kauf angeboten wird, die im Wirkungskreis des emittierenden kommunalen Unternehmens ansässig sind und die damit i.d.R. auch Kunden des Unternehmens sind. Durch die örtlich begrenzte Streuung der Bürgeraktien besteht zum einen die Möglichkeit, die Kundenbindung gezielt zu erhöhen, zum anderen kann dem Unternehmen durch die Emission der Bürgeraktien Kapital zufließen damit, z.B. im Bereich der Energieversorgung, ökologisch attraktive und rentable Projekte finanziert werden können. Dabei steht nicht nur der wirtschaftliche Erfolg des Unternehmens im Vordergrund, sondern auch der Lebensraum, für den sich die Bürger durch eine Kapitalbeteiligung engagieren können. Eine Verbesserung der Kapitalausstattung durch die Veräußerung von Bürgeraktien erhöht auch die Unabhängigkeit des kommunalen Unternehmens gegenüber der Haushaltslage der Trägerverwaltung. Damit einhergehend kann das kommunale Unternehmen seine finanzielle Eigenständigkeit und seine regionale Verbundenheit und Identiät stärken, erwirtschaftete Gewinne können in der Region investiert und müssen nicht an ein u.U. fernes, anonymes und unbeeinflussbares Mutterunternehmen abgeführt werden. 
Ein weiterer Aspekt des Konzepts der Bürgeraktie ist, der durch die Organisationsprivatisierungen herbeigeführten „Entpolitisierung“ entgegenzuwirken, auch wenn diese in den meisten Fällen durchaus erwünscht ist und den verstärkt betriebswirtschaftlich geprägten Entscheidungen der Unternehmensleitung entgegenkommt. Die Kapitalbeteiligung der Bürger an ,ihrem“ Kommunalversorger z.B. würde ihnen respektive ihren Vertretern im Aufsichtsrat wieder einen Zugang - mit Sitz und Stimme (!) - zu den nicht öffentlichen Sitzungen dieses Führungsorgans gewähren, ein Zugang, wie er in den Sitzungen des Kreistages oder des Gemeinderates nur als passiver Zuhörer möglich ist. Dies könnte zu einem steigenden Vertrauen der Bürger in das Management des kommunalen Unternehmens und evtl. auch zu einer Erhöhung der Transparenz des Unternehmens führen. Bei den Bestrebungen der Kommunalpolitik, mit Hilfe des so genannten Bürgerhaushalts dem Bürger die Kommunalpolitik näher zu bringen und Einfluss auf die Entscheidungen auszuüben, wird dies bereits vereinzelt praktiziert.

Dem Bürger wird mit seiner unmittelbaren Beteiligung an einer kommunalen AG die Möglichkeit gegeben, auf der Hauptversammlung von seinen Rechten als Aktionär Gebrauch zu machen, indem er z.B. als direkt Betroffener der Geschäftspolitik des Unternehmens hinterfragt oder dem Vorstand die Entlastung versagt oder eben nicht. Einflussmöglichkeiten eröffnet auch die Wahl der von der Hauptversammlung zu wählenden Eigentümervertreter in den Aufsichtsrat. Da eine kommunale Wahl in Angelegenheiten des kommunalen Unternehmens bei weitem nicht die Möglichkeiten gewährt wie eine Kapitalbeteiligung, kann das Konzept der Bürgeraktie der o.g. Entpolitisierung entgegenwirken und der jährlichen Hauptversammlung einen öffentlichen Charakter verleihen.

2. Bereits bestehende Konzepte der Bürgerbeteiligung

\section{a) Die Herten-Fonds der Stadtwerke Herten GmbH}

Die zu 100 Prozent der Stadt Herten gehörenden Stadtwerke Herten GmbH ${ }^{1}$ bieten ihren Stromkunden Anteile an den Stadtwerken in Form der sog. „Herten-Fonds“ an. Den potenziellen Anlegern werden Anteile an den Fonds in Höhe von $€ 1.000$,- bis $€ 20.000$,pro Person angeboten, die mit einer garantierten Verzinsung von 5\% p.a. verbunden sind. An diesen Fonds können sich sowohl private wie auch gewerbliche Stromkunden und Mitarbeiter der Stadtwerke Herten $\mathrm{GmbH}$ beteiligen. Sollten diese Kriterien nicht (mehr) erfüllt sein, müssen die Anteile wieder an die Stadtwerke zurückgegeben werden. Die durch die Beteiligung an den Fonds vereinnahmten Mittel fließen nach Angaben der Stadtwerke Herten GmbH nicht in den städtischen Haushalt, sondern in die Kasse der Stadtwerke zur Finanzierung von Projekten aus den Bereichen Versorgung und öffentliche Einrichtungen wie Bäder und Schulen. Anders als bei der Konzeption der

1 Vgl. Stadtwerke Herten GmbH (21. März 2004), http://www.hertener-stadtwerke.de. 
Bürgeraktie ist eine Mitbestimmung durch die Anteilseigner nicht möglich. Dafür werben die Stadtwerke Herten $\mathrm{GmbH}$ mit einer garantierten Verzinsung und der Finanzierung von Projekten.

\section{b) Elektrizitätswerke Schönau GmbH - EWS Schönau}

Die Elektrizitätswerke Schönau EWS $\mathrm{GmbH}^{2}$ (EWS) verfolgen eine stark ökologisch geprägte Zielsetzung mit ihrem Konzept der Bürgerbeteiligung. Den Kunden wird Strom aus kohle- und atomfreier Produktion angeboten. Des Weiteren wird die alternative Energieerzeugung durch bessere Einspeisevergütungen gefördert. Größtenteils gehören die EWS der Netzkauf Schönau GbRmbH, an der 750 Gesellschafter, i.d.R. Bürger aus Schönau, aber auch aus dem gesamten Bundesgebiet, und ca. 400 Beteiligte über den Schönauer Energiefonds der Gemeinschaftsbank Bochum beteiligt sind. Der Gesellschaftszweck der Netzkauf Schönau GbRmbH besteht darin, die EWS in die Lage zu versetzen, eine sichere und zugleich preisgünstige Stromversorgung zu betreiben. Die Umweltfreundlichkeit durch die Nutzung regenerativer Energiequellen und eine rationelle Energieumwandlung stehen dabei im Vordergrund. Zur Erreichung des Gesellschaftszwecks kaufte die Netzkauf Schönau GbRmbH das örtliche Stromnetz und gründete die EWS. In ihrer Rolle als Hauptgesellschafterin und durch einen gewählten Beirat beeinflusst die Netzkauf Schönau GbRmbH die Geschäftspolitik der EWS.

\section{Gemeinsamkeiten der Konzeptionen Herten-Fonds und EWS}

Die Konzepte der Versorgungsunternehmen Stadtwerke Herten GmbH und EWS basieren auf der gleichen Grundidee. In beiden Fällen wird das vom Bürger bzw. Kunden eingebrachte private Kapital zur Finanzierung von zuvor geplanten Projekten eingesetzt. Die Zielsetzung des Kapitalanlegers ist hingegen unterschiedlich: zum einen garantierte Verzinsung der Kapitaleinlage (Stadtwerke Herten), zum anderen ökologische Zwecksetzung (EWS). Eine konkretes Zielangebot ist auch für ein öffentliches Unternehmen unerlässlich bei dem Versuch, privates Kapital zur Anlage zu gewinnen.

\section{Voraussetzungen zur Bürgeraktienemission}

a) Gründung einer beteiligungsfähigen Rechtsform

Im Zuge der Umsetzung des Konzepts der Bürgeraktie als Beitrag zur Finanzierung kommunaler Unternehmen ist es notwendig, eine Rechtsform zu gründen, die die Beteiligung privater Dritter an einem kommunalen Unternehmen erlaubt bzw. überhaupt erst ermöglicht. Die Gründung einer solchen beteiligungsfähigen Rechtsform kann zum einen

2 Vgl. Elektrizitätswerke Schönau GmbH - EWS GmbH (21. März 2004), http://www.ews-schoenau.de. 
durch eine Ausgliederung zur Neugründung, zum anderen durch eine Sachgründung erfolgen. Da eine Sachgründung u.a. die Einzelübertragung aller Aktiva und Passiva in die AG erfordern würde, bietet die Ausgliederung zur Neugründung die praktikablere Möglichkeit. Nach den $\S \S 123$ Abs. 3 Nr. 2, 135, 168 ff. UmwG ${ }^{3}$ können die gesamten dem auszugliederndem Unternehmen zuzuordnenden Aktiva und Passiva im Rahmen der Gesamtrechtsnachfolge, im Sinne der $\S \S 171,131$ Abs. 1 Nr. 1 UmwG, auf die aufnehmenden Gesellschaft überführt werden. ${ }^{4}$ Die bei diesem Konzept favorisierte AG entsteht durch die sog. Organisationsprivatisierung bzw. formelle Privatisierung, bei der die kommunalen Aufgaben materiell noch immer unter staatlicher Verantwortung bleiben. ${ }^{5}$ Obwohl sich die Sach- und Neugründung voneinander unterscheiden, sind die Gründungsvorschriften der $\S \S 23-53 \mathrm{AktG}^{6}$ einzuhalten.

Neben den aktienrechtlichen Vorschriften müssen bei der Gründung von kommunalen Aktiengesellschaften die gemeinderechtlichen Regelungen beachtet werden. Die in den Gemeindeordnungen der Flächenbundesländer festgelegten Vorschriften für die wirtschaftliche Betätigung von Gebietskörperschaften (Gemeindewirtschaftsrecht) regeln, unter welchen Umständen und Voraussetzungen die Kommunen wirtschaftliche und nichtwirtschaftliche Unternehmen in privatrechtlicher Form errichten, erweitern und sich an ihnen beteiligen können. ${ }^{7}$ Die kommunalrechtlichen Vorschriften besagen, dass das Unternehmen durch den öffentlichen Zweck gerechtfertigt und für seine Erfüllung erforderlich sein muss, dass es in seinem Umfang und seiner Art in einem angemessenen Verhältnis zum Bedarf und der Leistungsfähigkeit der Kommune steht und den öffentlichen Zweck besser oder wirtschaftlicher als ein anderer privater Dritter erfüllt. Der Hauptzweck des Unternehmens darf nicht die Gewinnmaximierung sein, wobei das Abwerfen eines Ertrags für den Gemeindehaushalt als Nebenzweck durchaus erlaubt ist. ${ }^{8}$

3 Umwandlungsgesetz - UmwG vom 28. Oktober 1994, BGBl. I 1994 S. 3210, BGBl. I 1995, S. 428, zuletzt geändert durch Gesetz vom 09. Dezember 2004, BGB1. I 2004, S. 3214.

4 Vgl. Hübner (2004), S. 54.

5 Vgl. Ronellenfitsch (2004), S. 25.

6 Aktiengesetz - AktG vom 06. September 1965, BGBl. I 1965, S. 1089, zuletzt geändert durch vom 15. Dezember 2004, BGB1. I 2004, S. 3408.

7 Vgl. Art. 86-96 GO BY v. 22. August 1998, GVBl., S. 797 ff., zuletzt geändert durch Gesetz vom 24. Dezember 2002, GVB1., S. 962; $\S \S 100-110$ GO BR v. 15. Oktober 1993, GVB1. I, S. 398, zuletzt geändert durch Gesetz v. 04. Juni 2003, GVB1. I 2003, S. 172, 174; §§ 102-108 GO BW v. 24. Juli 2000, GVB1., S. 582, zuletzt geändert durch Gesetz v. 28. Mai 2003, GVBl., S. 271; §§ 121-127c GO HE v. 01. April 1993, GVB1. I, S. 534, zuletzt geändert durch Gesetz v. 20. Juni 2002 GVB1. I, S. 342; §§ 68-77, 122 KVerf MV v. 13. Januar 1998, GVOB1. MV, S. 29, ber. S. 890, zuletzt geändert durch Gesetz v. 26. Februar 2004, GVOBl, S. 61; $\S$ 108-116 GO NI v. 22. August 1996, GVB1., S. 382, zuletzt geändert durch Gesetz v. 12. Dezember 2003, GVBl., S. 446; $\S 107-115$ GO NW v. 14. Juli 1994, GV. NW, S. 666 ff., zuletzt geändert durch Gesetz v. 29. April 2003, GV. NW, S. 254; $\S 85-92$ GO RP. v. 31. Januar 1994, GVB1., S. 153, zuletzt geändert durch Gesetz v. 21. Juli 2003, GVBl., S. 155, 158; §§ 108-118 KSVG SL v. 27. Juni 1997, Amtsbl. S. 682, zuletzt geändert durch Art. 1 d. Gesetzes v. 08. Oktober 2003, Amtsbl. S. 2874; §§ 95-102 GO SN v. 18. März 2003, GVB1., S. 55, berichtigt durch Gesetz v. 25. April 2003, GVB1. S. 159; $\S$ 116-124 GO ST v. 05. Oktober 1993, GVB1., S. 568, zuletzt geändert durch Gesetz v. 26. März 2004, GVB1., S. 234); §§ 101-109 GO SH v. 28. Februar 2003, GVOB1. S. 57; §§ 71-77 KommunalO TH v. 28. Januar 2003, GVB1. S. 41.

8 Vgl. Gern (2003), S. 460. 
b) Statuierung des öffentlichen Zwecks

Trotz des ohnehin elementaren Charakters, der der Satzungsgestaltung im Gründungsprozess von AGen zugesprochen wird, gewinnt dieser noch einmal an Bedeutung wenn dies im Zusammenhang mit der wirtschaftlichen Betätigung von Gebietskörperschaften erfolgt. ${ }^{9}$ Die Satzung ist eine wesentliche Grundlage für die Arbeit der Organe der AG, für deren Ausgestaltung die oder der Gründer der Gesellschaft verantwortlich sind. ${ }^{10}$ Die Gemeinde muss in ihrer Rolle als Unternehmensträgerin und, zunächst noch, Alleinaktionärin den ihr zustehenden Einfluss auf die Ausgestaltung der Statuten der AG geltend machen und damit die Arbeit der Organe an den Inhalt der Satzung binden. ${ }^{11}$ Nach Maßgabe der meisten Gemeindeordnungen ist daher der öffentliche Zweck in der Unternehmenssatzung als Unternehmensziel festzulegen. ${ }^{12}$ Der Inhalt der Satzung muss zudem derart formuliert sein, dass auf eine Gewinnmaximierung explizit verzichtet wird und die Verfolgung des öffentlichen Zwecks als oberstes Unternehmensziel festgelegt wird. Damit wird der befürchteten Flucht aus den öffentlich-rechtlichen Bindungen des Kommunalrechts in das Privatrecht entgegengesteuert. ${ }^{13}$ Die Existenzberechtigung privatrechtlicher Unternehmen der öffentlichen Hand wird mithin durch die Verfolgung des öffentlichen Zwecks begründet. ${ }^{14}$ Bei der Sicherstellung der Verfolgung des öffentlichen Zwecks ist daher die Satzung der kommunalen AG das wichtigste Steuerungsinstrumentarium für die Kommune. Nur durch die Verankerung des Unternehmenszwecks in der Unternehmensverfassung und der Festlegung der Einflussmöglichkeiten des Aufsichtsrats und der Hauptversammlung kann die Kommune den aktienrechtlichen Freiheiten des Vorstands in ihrem Sinne entgegenwirken. ${ }^{15}$

Ein weiterer, nicht den kommunalrechtlichen Regelungen entspringender Grund ist der Schutz der Anleger. Dem potenziellen Bürgeraktionär muss klar sein, dass das Unternehmen primär öffentliche Zwecke verfolgt und nicht zur Gewinnmaximierung angehalten ist. Durch die Gründung von Unternehmen in einem profitablen Wirtschaftszweig wie etwa der Energieversorgung können die Verlustrisiken zwar verringert werden, dennoch ist das Unternehmen der Verfolgung des öffentlichen Zweck verpflichtet und nicht der Gewinnmaximierung. Dies führt zu widerstreitenden Interessen bei dem Aktionär als Anleger und dem Aktionär als Bürger/Gebührenzahler. ${ }^{16}$

9 Vgl. Raiser (2001), S. 84.

10 Vgl. § 2 AktG.

11 Vgl. Ade (1997), S. 117.

12 Vgl. §103 Abs. 1 Satz 1 Nr. 2 GO BW; § 69 Abs. 1 Nr. 2 KVerf MV; § 109 Abs. 1 Nr. 5 GO NI; § 108 Abs. 1 Satz 1 Nr. 7 GO NW; $\S 87$ Abs. 1 Satz 1 Nr. 2 GO RP; § 117 Abs. 1 Nr. 2 GO ST; Art. 92 Abs. 1 Satz 1 Nr. 1 GO BY.

13 Vgl. Püttner (1985), S. 83.

14 Vgl. Ade (1997), S. 117.

15 Vgl. Kröger (2001), S. 31.

16 Vgl. auch Harbarth (1998), S. 42 ff. 


\section{c) Kapitalerhöhung und Aktienemission}

Neben der Gründung einer AG als Voraussetzung für die Emission von Bürgeraktien bedarf es einer ordentlichen Kapitalerhöhung ${ }^{17}$ zur Erweiterung der Kapitalbasis des Unternehmens und zur Sicherstellung des beabsichtigten Finanzierungseffekts. Die im Zuge der Kapitalerhöhung geschaffenen neuen Aktien sind an die potenziellen Bürgeraktionäre zu emittieren. Dies kann durch Selbstemission geschehen: Die AG bedient sich dabei keines Emissionskonsortiums, das i.d.R. aus mehreren Kreditinstituten besteht und ein weites Vertriebsnetz für Wertpapiere besitzt, sondern emittiert die neuen Aktien auf eigenes Risiko und auf eigene Rechnung an einen von vornherein begrenzten Personenkreis bzw. durch den Wirkungskreis des kommunalen Unternehmens örtlich begrenztem Radius. Die Aktiengattung muss ebenfalls festgelegt werden. Wenn das Unternehmen die Aktionärsstruktur beobachten und steuern will, ist es ratsam, vinkulierte Namensaktien zu emittieren. Da die Übertragung solcher Aktien der Zustimmung des Unternehmens bedürfen, kann die Steuerung des Aktionärskreises sichergestellt und das Unternehmen vor eventuellen Übernahmen durch andere Unternehmen geschützt werden. ${ }^{18}$ Aufgrund des von vornherein definierten Personenkreises empfiehlt sich für das kommunale Unternehmen die Privatplatzierung, um den Bürgern die Aktien zum Kauf anzubieten. Die Privatplatzierung bietet der AG zusätzlich die Möglichkeit, ein Gleichgewicht zwischen der angebotenen und nachgefragten Menge der Aktien zu schaffen. ${ }^{19}$

\section{Die Bedeutung der Bürgeraktie für die beteiligten Akteure}

Die am Konzept der Bürgeraktie beteiligten Akteure haben unterschiedliche Motive und Interessen, die für eine Realisierung der Konzeption von großer Bedeutung sind. Als Akteure treten die Trägerverwaltung als Allein- bzw. Hauptaktionärin, das kommunale Unternehmen in Form einer AG als Emittent der Bürgeraktien und die Bürger als potenzielle Anleger auf den Plan. Aufgrund der unterschiedlichen Stellungen der Akteure gibt es je nach Sicht unterschiedliche Einschätzungen der Bedeutung der Bürgeraktie.

1. Die Bedeutung der Bürgeraktie für die Trägerverwaltung als (Haupt-)Aktionärin des kommunalen Unternehmens

Als Folge der Organisationsprivatisierung wird die Trägerverwaltung in ihren Einflussmöglichkeiten auf das kommunale Unternehmen durch das Gesellschaftsrecht, in diesem Fall das AktG, beschränkt. Gegebenenfalls sind einschlägige Mitbestimmungs-

17 Kapitalerhöhung gegen Einlagen, $\S$ 182-191 AktG.

$18 \S 68$ AktG, vgl. auch Perridon/Steiner (2002), S. 369; Olfert/Reichel (Hrsg.) (2003), S. 212; Bieg/Kußmaul (2000), S. 104; Gräfer/Beike/Scheld (2001), S. 122.

19 Vgl. Gerke/Rapp (1993), S. 306. 
gesetze $^{20} \mathrm{zu}$ beachten, die die personelle Vertretung der Eigentümerseite im Aufsichtsrat weiter schmälern. Die Ausgabe von Bürgeraktien führt automatisch dazu, dass der Einfluss der Trägergemeinde, vertreten durch den Gemeindevorstand, dieser wiederum vertreten durch die von ihm entsandten Vertreter, abnimmt, da die Bürgeraktionäre auf der Hauptversammlung eigene Vertreter zur Wahl in den Aufsichtsrat stellen können.

2. Die Bedeutung der Bürgeraktie für das kommunale Unternehmen

Das kommunale Unternehmen, das die Bürgeraktien emittiert, sieht in der Bürgerbeteiligung in erster Linie die Möglichkeit, frisches Kapital durch die Kapitalerhöhung in das Unternehmen fließen zu lassen. Eine verbesserte Kapitalausstattung kann dem Unternehmen für notwendige Ersatz-, Modernisierungs- und Erweiterungsinvestitionen dienen. Des Weiteren führt eine verbesserte Ausstattung mit Kapital zu einer höheren Kreditwürdigkeit bei Banken. Angesichts der Tatsache, dass eine nicht insolvenzfähige Gebietskörperschaft am Unternehmen beteiligt ist, ist das Argument der Kreditwürdigkeit zwar nachrangig; gleichwohl ist angesichts der unterdurchschnittlichen Eigenkapitalquote vieler öffentlicher Unternehmen und mit Blick auf die gestiegenen Anforderungen in Folge des Basel II-Akkords eine Aufstockung des haftenden Kapitals empfehlenswert. Die Bürgerbeteiligung am kommunalen Unternehmen kann als Nebeneffekt zur Finanzierung auch zu einer Erhöhung der Kundenbindung führen, da sich die Bürgeraktionäre mit ihrem kommunalen Unternehmen und dessen Leistungen, z.B. dem Betrieb von Bädern oder Anlagen zur ökologischen Energiegewinnung aus regenerativen Energien, identifizieren können. Neben der Kundenbindung bedeutet die Bürgeraktie für das Unternehmen, wie bereits erwähnt, eine örtliche Begrenzung des Aktionärskreises und, damit verbunden, ein eventuell geringerer Emissionserlös als dies z.B. bei dem Gang an die Börse oder bei der Veräußerung von Aktien an einen Investor der Fall wäre.

\section{Die Erwartungen der Bürgeraktionäre}

Neben der Bedeutung der Bürgeraktie für die Trägerverwaltung und das kommunale Unternehmen messen die potenziellen Bürgeraktionäre ihrer Kapitalbeteiligung eine gewisse Bedeutung bei. Sie versprechen sich eventuell ein Mitspracherecht bei der Verwendung der Gewinne und bei Änderungen der Satzung, die einer 3/4-Mehrheit bedürfen. Je nach Ausgestaltung der Satzung können die Aktionäre, neben der Verwaltung nun auch die Bürger, ihre Stimmen für oder gegen bestimmte, von der Hauptversammlung zu genehmigende Beschlüsse oder Geschäfte, einsetzen. Diese Form der Mitsprache - z.B.

20 Gesetz über die Mitbestimmung der Arbeitnehmer in den Aufsichtsräten und Vorständen der Unternehmen des Bergbaus und der Eisen und Stahl erzeugenden Industrie (Montan-Mitbestimmungsgesetz - MontanMitbestG) vom 21. Mai 1951, BGB1. I 1951, S. 347; Gesetz über die Mitbestimmung der Arbeitnehmer (Mitbestimmungsgesetz - MitbestG) vom 04. Mai 1976, BGB1. I 1976, S. 1153, zuletzt geändert durch Gesetz vom 18. Mai 2004, BGB1. I 2004, S. 974; Gesetz über die Drittelbeteiligung der Arbeitnehmer im Aufsichtsrat (Drittelbeteiligungsgesetz - DrittelbG) vom 18. Mai 2004, BGB1. I 2004, S. 974. 
bei der Eröffnung neuer oder dem Abstoßen bestehender Geschäftsfelder, der Konzernbildung, der Kapitalerhöhung zur institutionalisierten Absicherung eines PrivatePublic-Partnerships - folgt der bereits erwähnten Idee, die Bürger und letztlich auch die Konsumenten der vom Unternehmen produzierten Produkte und Dienstleistungen an den in die privatrechtliche Sphäre verlagerten Entscheidungen über das Gemeinwohl und die Daseinsvorsorge teilhaben und mitbestimmen zu lassen. Dem Bürgeraktionär dürfte im Zweifel mehr daran gelegen sein, die örtlichen öffentlichen Einrichtungen wie Schwimmbäder und Ver- und Entsorgungseinrichtungen zu sanieren als Prestigeobjekte wie Messeund Kongresszentren oder Hotels in der Kommune zu errichten.

Das Konzept der Bürgeraktie kann dem bereits erwähnten Streben nach Transparenz von Seiten der Bürger erhöhen. Der Kunde und Aktionär des kommunalen Unternehmens ist an einem „durchsichtigen“ Management interessiert. Da öffentliche Sitzungen durch die nichtöffentlichen Sitzungen der Organe der AG ersetzt werden, bleibt dem Bürger/Aktionär/Kunden nur die Kapitalbeteiligung in Form der Bürgeraktie als Zugang zur Hauptversammlung.

Der Anleger, der einen Teil seines Vermögens in die Aktien eines Unternehmens investiert, erhofft sich allerdings regelmäßig auch eine angemessene Verzinsung seines Kapitals. Damit Dividenden an die Anteilseigner ausgeschüttet werden können, müssen Gewinne durch den Absatz der produzierten Produkte und Dienstleistungen erwirtschaftet werden. Damit aus einem potenziellen Bürgeraktionär ein richtiger Bürgeraktionär wird, müssen die Anreize, die zum Kauf der Aktien geschaffen werden, über die bloße Verzinsung hinausgehen (die der Aktionär als Kunde ohnehin über entsprechende Preise mitbezahlt). Das heißt, dass die Beteiligungsquote der Bürger bei mind. 25\% plus einer Aktie liegen sollten. Hinzu kommt, dass dieser Anteil des Grundkapitals auf der Hauptversammlung anwesend sein muss, damit es zur erfolgreichen Einflussnahme bei Grundsatzentscheidungen, die einer $3 / 4$-Mehrheit bedürfen, kommt. Ist z.B. in der Satzung festgelegt, dass die 3/4-Mehrheit der Hauptversammlung nötig ist um über Entgeltanpassungen für Produkte und Dienstleistungen des Unternehmens oder die Einschränkung des Angebots von Dienstleistungen, z.B. der ÖPNV oder Einrichtungen wie Bäder, zu beschließen, kann die Attraktivität einer Kapitalbeteiligung über das rein finanzielle Interesse in Gestalt einer Dividende hinausgehen. Die Betroffenen könnten somit in die Entscheidungen des Unternehmens eingebunden werden und einen Beitrag zur Entpolitisierung durch die Verlagerung kommunaler Verantwortung aus dem öffentlich-rechtlichen Bereich in die Sphären des Privatrechts leisten - die Kommunalpolitiker als Mittler zwischen individuellen Bürgerinteressen und kollektiven Notwendigkeiten bzw. Möglichkeiten verlieren an Bedeutung. 


\section{Zu erwartende Konfliktpotenziale bei der Beteiligung von Bürgern an kommunalen Unternehmen}

\section{Der Bürger in seiner Rolle als Aktionär und Kunde des kommunalen Unternehmens}

Wird dem Bürger ein wirksamer Einfluss auf das Unternehmen durch die Hauptversammlung eingeräumt, stellt sich die Frage, wie der Bürger in seiner Rolle als Aktionär und Kunde des kommunalen Unternehmens abstimmen wird, wenn Entscheidungen anstehen, die sowohl die Belange des Bürgers als auch die des an Gewinnen interessierten Anlegers betreffen. Als Kunde des Unternehmens, der die vom Unternehmen angebotenen Ver- und Entsorgungsleistungen durch den Anschluss- und Benutzungszwang in Anspruch nimmt, ist der Bürger dazu geneigt Entscheidungen zu favorisieren, die eine nachhaltige Preis- oder Gebührensenkung zu Folge haben. Er würde demnach einer auf der Hauptversammlung zur Disposition stehenden Investitionsentscheidung zustimmen, wenn sich diese direkt positiv auf seinen Lebensstandard auswirkt. Ist der Bürger jedoch stärker an einer angemessenen Verzinsung seiner Anlage interessiert, kann er dazu geneigt sein, seine Stimme auf der Hauptversammlung zugunsten von gewinnbringenden Investitionen abzugeben.

Dieses Dilemma kann durchaus erwünschte Folgen haben, da der fordernde Bürger gleichzeitig die betriebswirtschaftlichen Nebenbedingungen bzw. Wirkungen zu spüren bekommt und insofern genau die Abwägungen vorzunehmen hat, die sonst Aufgabe des Politikers sind - der Bürger erkennt die „Schwierigkeiten des Geschäfts“.

2. Wahrung der Steuerungs- und Einflussmöglichkeiten auf das kommunale Unternehmen durch die Trägerverwaltung

Der Erhalt des Einflusses der Verwaltung auf die Aktiengesellschaft durch die Hauptversammlung sinkt durch die Veräußerung von Anteilen an die Bürger. Schon bei einer Beteiligungsquote von $25 \%$ plus einer Aktie ist es den Bürgern möglich, Grundsatzentscheidungen, die einer 3/4-Mehrheit bedürfen, auf der Hauptversammlung zu blockieren. Sollte die Verwaltung bereit sein, auf ihre 3/4-Mehrheit zu verzichten, muss sie die Statuten der Gesellschaft ändern, die andere Mehrheitsverhältnisse, z.B. die einfache Mehrheit bei zustimmungspflichtigen Geschäften, zulassen. Der Verwaltung bleibt als Steuerungsinstrument demnach nur die Satzung der AG. Diese gilt es zum eigenen Vorteil auszugestalten, wenn die Kommune weiterhin ihren Einfluss auf die AG aufrechterhalten will. 


\section{Ergebnis}

Wie die Überlegungen zeigen, ist die Beteiligung von Bürgern an kommunalen Unternehmen, die als AG geführt werden, grundsätzlich möglich, da es sich um eine Beteiligung privater Dritter handelt und diese nach gemeinderechtlichen Regelungen grundsätzlich zulässig ist. Die Bürgeraktie im Zuge einer Kapitalerhöhung zu emittieren, kann einen sinnvollen Beitrag zur Finanzierung kommunaler Unternehmen leisten - die Eingangsfrage wäre also zu bejahen.

Die Bedeutung der Bürgeraktie geht über den rein finanziellen Aspekt jedoch weit hinaus.

Die Politik bzw. Verwaltung der Gebietskörperschaft müssen entscheiden, welche Zugeständnisse sie den neuen Bürgeraktionären einräumt. Da das kommunale Unternehmen auf die Erfüllung des öffentlichen Zwecks ausgerichtet ist und die Gewinnmaximierung als nachrangig gilt, kann die monetäre Attraktivität der Bürgeraktie darunter leiden. Den potenziellen Bürgeraktionären muss die finanzielle Beteiligung an der kommunalen AG demnach erstrebenswert gemacht werden. Dies kann über das monetäre Interesse der Bürger (Renditeerwartungen) ohne Mitsprachemöglichkeiten geschehen. Es ist aber genauso denkbar, dass die - vermeintlichen - Einflussmöglichkeiten zu einer Anlage führen.

Bürgeraktien führen zu einer Entpolitisierung und Versachlichung von Entscheidungen. Die Politik und ihre Verwaltung verlieren an Einflussmöglichkeiten, gleichzeitig werden die der Bürger gleich Aktionäre gleich Kunden gestärkt. Dieser Effekt ist in seiner Bedeutung gar nicht zu überschätzen, wie die vielfach erbitterten Diskussionen in zahlreichen Gemeinden, Städten und Kreisen zur Zukunft ihrer kommunalen Unternehmen zeigen. Wollen die Bürger wirklich die bisher gemeindliche Abfallsammlung an einen Zweckverband oder Privaten abgeben (wie die Politik), oder wollen die Bürger wirklich den Verkauf des bisher stadteigenen Wasserwerks an einen international aufgestellten Konzern (wie die Politik)? Solche zurzeit bundesweit gestellten Fragen würden von Bürgeraktionären wohl oft anders als von der Politik beantwortet werden.

\section{Literaturverzeichnis}

Ade, Klaus (Hrsg.) (1997), Handbuch Kommunales Beteiligungsmanagement, Stuttgart

Bieg, Hartmut und Heinz Kußmaul (2000), Investitions- und Finanzierungsmanagement, München

Elektrizitätswerke Schönau GmbH - EWS GmbH (21. März 2004), http://www.ews-schoenau.de

Gerke, Wolfgang und Heinz-Werner Rapp, (1993), Eigenkapitalbeschaffung durch Erstemission von Aktien, in: Handbuch des Finanzmanagements - Instrumente und Märkte der Unternehmensfinanzierung, hrsg. von Günther Gebhardt, Wolfgang Gerke und Manfred Steiner, München, S. 287-312

Gern, Alfons (2003), Deutsches Kommunalrecht, 3. Aufl., Baden-Baden

Gräfer, Horst, Rolf Beike und Guido A. Scheld (2001), Finanzierung - Grundlagen, Institutionen, Instrument und Kapitalmarkttheorie, 5. Aufl., Berlin

Harbarth, Stephan (1998), Anlegerschutz in öffentlichen Unternehmen - Untersuchungen über das Spar-, Giro- und Kreditwesen: in: Schriften des Instituts für deutsches und internationales Recht des Spar-, Giro- und Kreditwesens an der Johannes Gutenberg-Universität Mainz; Bd. 122, hrsg. von Walther Hadding und Uwe H. Schneider, Berlin

Hübner, Heinrich (2004), Beteiligung eines Investors an kommunalen Betrieben, in: KommJur, Heft 2, S. 54-57 
Kröger, Christian W. (2001), Kommunale Sonderfinanzierungsformen - Möglichkeiten und Grenzen einer Börseneinführung von Versorgungsunternehmen, Wiesbaden

Olfert, Klaus und Christopher Reichel (Hrsg.) (2003), Finanzierung, 12. Aufl., Ludwigshafen

Perridon, Louis und Manfred Steiner (2002), Finanzwirtschaft der Unternehmung, 11. Aufl., München

Püttner, Günter (1985), Die öffentlichen Unternehmen - Ein Handbuch zu Verfassungs- und Rechtsfragen der öffentlichen Wirtschaft, 2. Aufl., Stuttgart, München und Hannover

Raiser, Thomas (2001), Recht der Kapitalgesellschaften - Ein Handbuch für Praxis und Wissenschaft, 3. Aufl., München

Ronellenfitsch, Michael (2004), Neue Privatisierungsdiskussion, in: Handbuch Kommunale Unternehmen, hrsg. von Werner Hoppe und Michael Uechtritz, Köln, S. 15-27

Stadtwerke Herten GmbH (21. März 2004), http://www.hertener-stadtwerke.de 